\title{
The Effects of a Skin Cancer Educational Intervention on Beliefs, Knowledge and Behaviours of Outdoor Workers in the Tropics
}

\author{
Lesley E. Paine and Marie L. Caltabiano \\ James Cook University-Cairns
}

\begin{abstract}
Recent research has linked some forms of skin cancer with ultraviolet radiation from constant sun exposure. Outdoor workers represent an 'at risk' group as daily exposure to the sun's harmful rays is a necessary part of their occupations. This study assessed changes in beliefs, knowledge and behaviours of outdoor workers in North Queensland towards skin cancer. A total of 40 outdoor workers participated in the study. A quasi-experimental repeated measures design with a treatment and control group was used. Subjects in the treatment group were exposed to an educational intervention explaining the danger of skin cancer and ways that the disease could be prevented. Results indicated that in comparison to the control group, subjects who received the educational intervention reported greater levels of sun protection behaviour, had increased knowledge about the disease and reported changes in their beliefs concerning skin cancer. Changes were assessed by using a sun safety questionnaire. Results of this research are of value to the Queensland Cancer Fund, Government Health Promotion Departments and numerous companies that employ outdoor workers in the tropics.

Skin cancer has become a serious health concern in Australia where the reported incidence of the disease is the highest in the world (AIHW, 1998; Arthey \& Clarke, 1995). There are two types of skin cancer - melanomas and non-melanomas. Melanoma is the rarest, yet most dangerous form of skin cancer. It can appear at any age and on any area of the body, not only those exposed to the sun. It is often fast growing and, if left untreated, can be carried quickly to distant parts of the body to form secondary cancers. The first sign of a melanoma is usually a change in a freckle or mole, or the appearance of a new spot on normal skin. There may be a change in size, shape or colour of a spot and the surface may become raised (National Health \& Medical Research Council, 1996; Queensland Cancer Fund, 1998). Early detection of melanoma is vital as in $95 \%$ of cases it can be surgically removed and the person completely cured (Borland, 1979; Hill et al., 1993).
\end{abstract}


In addition to melanocytic skin cancers, there are two types of non-melanocytic skin cancers; basal cell carcinomas (BCCs) and squamous cell carcinomas (SCCs). BCCs are the most common, but the least dangerous type of skin cancer, accounting for $75 \%$ of skin cancers in Australia. They may appear as a pale lump, a small ulcer that does not heal, or a reddish flaky patch (Arthey \& Clarke, 1995; Cody \& Lee, 1990). In contrast, SCCs are less common than BCCs accounting for 15 to 20 percent of reported skin cancers. They grow more rapidly than BCCs and may spread to other parts of the body if left untreated. Approximately 1 to 2 percent of SCCs spread and become life threatening (Queensland Cancer Fund, 1998). This form of skin cancer can look like a thickened, scaly spot which may bleed or ulcerate, and occurs predominantly in people over the age of 50. This suggests that they are a result of years of exposure to the sun (Emmett, 1988).

Apart from these cancerous skin spots there are also non-cancerous solar keratoses, commonly known as sunspots. Although sunspots are not skin cancers, they are a warning sign that the skin is of the type that is prone to developing skin cancer (Queensland Cancer Fund, 1998). People who develop solar keratoses should take extra care in the sun and regularly examine their skin for new spots (Emmett, 1988). Studies examining skin cancer in outdoor workers have found that they report high levels of solar keratoses, pointing to a need for sun protective behaviours in this group (Borland et al., 1994; English, 1996).

\section{Prevention of Skin Cancer}

In Australia, the primary prevention of skin cancer has focused on reducing personal exposure to the known risk factors for the disease through the use of sun protective behaviours, skin examination and treatment seeking (Cody \& Lee, 1990; Queensland Cancer Fund, 1997a). Research has indicated that the most important sun protective behaviour is to avoid exposure to the sun between 10 am and $2 \mathrm{pm}$. The sun's rays are the most intense during these hours of the day, and by avoiding direct exposure to it, an individual can greatly reduce the risk of sun damage to their skin (Cody \& Lee, 1990).

With regard to sun safety, workplace health and safety standards state that employers have a duty of care to minimise employees' exposure to the sun by designing and providing equipment that will protect the employee from ultra violet radiation. Similarly, employees have a duty of care to use any equipment given to prevent the development of sun-related 
diseases and to obey instructions given by employers regarding sun protection (Queensland Cancer Fund, 1999b).

Although the best form of protection is to avoid sun exposure, for some people like outdoor workers in the tropics, avoiding the sun during these hours of the day is virtually impossible. In these cases clothes provide the best possible protection. In places where the skin is not covered by clothing, sunscreen can be used for protection. This sunscreen should be a broad-spectrum one, with a sun protection factor (SPF) of 15 or higher, and should be reapplied every 2 hours (or more frequently if perspiring) (Marks, 1996). It is important to note that even if these sun protective behaviours are followed, regular skin examination is still required to ensure that there are no changes in existing freckles or moles that may be caused by skin cancer (National Health \& Medical Research Council, 1996; Queensland Cancer Fund, 1997b).

Skin cancer education can be an effective mechanism for conveying information about the disease, warning signs, and sun-protective behaviours. When used in conjunction with skin examination and treatment seeking, education can be a powerful force in the prevention of skin cancer. Borland, Hill and Noy (1990) reported an increased awareness of sun protection messages following a skin cancer prevention program. Similarly, Jones and Leary (1994) found that sun protection behaviour increased as a result of a skin cancer education program which emphasised the negative effects of the sun on physical appearance. Another study by Buller et al. (1994) aimed to increase knowledge about the effects of sun exposure, skin cancer and prevention in a school setting by introducing skin cancer education. Results showed that the intervention was more effective in influencing knowledge and attitudes than in changing behaviour.

Research on outdoor workers has also indicated beneficial effects of skin cancer education. In a study by Borland et al. (1991) which examined the impact of a skin cancer education package for outdoor workers in Victoria, sun protective behaviours were found to increase following the program. It should be noted that Borland et al.'s (1991) intervention was carried out on workers in a cooler climate with softer sunlight than the tropics. Outdoor workers in tropical areas such as North Queensland represent a serious 'at risk' group. Educating these workers about the danger of skin cancer, and teaching them sunprotective behaviours, should help to raise their awareness and concerns about the disease, and motivate them to engage in preventive behaviours. 
The present study was designed to assess changes in beliefs, knowledge and behaviours of outdoor workers towards skin cancer following a sun safety education session explaining the danger of skin cancer and ways that the disease can be prevented. A controlled pre-post-test design was used to test the effectiveness of the intervention. For ethical reasons, upon completion of the study the educational intervention was administered to subjects in the control group.

The hypotheses tested were:

1. The treatment group will report higher levels of sun protection behaviour than they did at the start of the study. The treatment group will report more sun protection behaviours than the control group.

2. At the conclusion of the study outdoor workers in the treatment group will report more knowledge about skin cancer, than those in the control group.

3. The beliefs of outdoor workers in the treatment group will change between the pre and post-test due to the educational intervention.

4. Outdoor workers in the treatment group but not those in the control group, will reduce their amount of sun exposure on weekends.

\section{Method}

\section{Subjects}

Forty male subjects participated in this study. Their average age was 32 years with a standard deviation of 9.4 years. Participants ranged in age from 18 to 60 years. These subjects were convenience sampled from two construction/maintenance companies that employed outdoor workers. Occupations of the subjects included builders, bricklayers, electricians, roof tilers, trades assistants and plumbers. The workers had not previously been exposed to sun safety talks in their workplace.

The majority of the subjects who participated in the study had completed a trade certificate at Tafe (80\%) and were born in Australia (92.5\%). The length of employment as an outdoor worker varied, however, $40 \%$ reported being employed for 10 or more years. 


\section{Skin Cancer Education (Intervention)}

A quasi-experimental design with an intervention and control group was used, as the prerequisite of random assignment of participants to groups (in this case companies) could not be met. The assignment of a company to either the intervention or the control group was determined by the toss of a coin. The intervention and control groups were administered questionnaires on separate days. Each group of subjects were measured before and after the intervention (pre and post-tested).

The intervention was developed using guidelines set by the Queensland Cancer Fund. The educational talk about skin cancer prevention in outdoor workers had six components:

1. What is skin cancer?

2. Who is "at risk" of developing skin cancer?

3. Why Australia and tropical North Queensland?

4. How is the disease prevented?

5. Why is skin examination and treatment seeking so important?

6. Question and answer session.

Handouts to accompany the talk were given to the subjects.

\section{Questionnaire}

The self-report questionnaire was constructed by staff at the Queensland Cancer Fund and the Sunshine Coast Regional Health Authority. Demographic information was obtained on gender, age, education level, occupation and birth origin. Five questions assessed beliefs about skin cancer and four questions assessed knowledge about skin cancer. Information was also gathered on skin colour and average time spent in the sun. The remaining questions focused on sun protection behaviours, skin examination, treatment seeking and incidence of skin cancer.

One month after administering the first questionnaire, the sun safety talk was given to the intervention group. The principal researcher had undertaken a skin cancer educator's course through the Queensland Cancer Fund. Employees were allowed to ask questions about anything they were unsure of. 
Five weeks after the sun safety talk was given, the intervention and control group were administered the questionnaire again by the researchers, to assess any changes that may have occurred and to measure the effectiveness of the intervention. It is important to note that the treatment group were not aware that there was a control group and vice versa.

\section{Results}

\section{Descriptive Statistics}

Of the subjects who participated in the study $12.5 \%$ reported never being sunburnt, $20 \%$ being sunburnt once, 35\% 2-5 times and 32.5\% 6 times or more. Forty percent of subjects reported having sunspots before the intervention and 55\% after the intervention. One person in the study reported that they had suffered a melanoma.

Prior to the educational intervention, $50 \%$ of subjects in the treatment group reported that they spent 7 hours a day in the sun on the weekdays $(\underline{\mathrm{M}}=5.25$; $\mathrm{SD}=.91$; Range of scores: $1=$ less than 30 minutes a day, and 5= more than 7 hours a day), whereas after the intervention there was only a slight decrease to $45 \%(\underline{M}=5 ; \mathrm{SD}=1.08)$. In contrast, $20 \%$ of subjects in the control group reported spending 7 hours a day in the sun on the pre-test $(\underline{\mathrm{M}}=4.3 ; \mathrm{SD}=$ 1.34) and similar on the post-test $(\underline{\mathrm{M}}=4.15 ; \mathrm{SD}=1.39)$.

Subjects in the treatment group reported spending slightly less time in the sun on the weekends for the post-test $(\underline{\mathrm{M}}=2.65, \mathrm{SD}=.67)$ than compared to the pre-test $(\underline{\mathrm{M}}=4.2, \mathrm{SD}=$ 1.15). For the control group the reported time spent in the sun on the weekends was similar for the pre-test $(\underline{M}=4.15 ; \mathrm{SD}=1.09)$ and the post-test $(\underline{M}=4.1 ; \mathrm{SD}=1.16)$.

Table 1 gives the reported sun protection behaviours before and after the intervention. Of the subjects in the study who reported using sunscreen, 15 people used a sunscreen with a sun protection factor (SPF) of $15+, 1$ person used a sunscreen with an SPF of 30+, 1 person used a sunscreen with a SPF of 30 and 2 people used a sunscreen with an SPF of 15.

\section{Table 1}

\section{Percentage of outdoor workers reporting sun protection behaviours before and after the intervention.}




\begin{tabular}{|c|c|c|c|c|}
\hline \multirow{3}{*}{$\begin{array}{l}\text { Sun Protection } \\
\text { Behaviour }\end{array}$} & \multicolumn{2}{|c|}{ Treatment $(\mathrm{N}=20)$} & \multicolumn{2}{|c|}{ Control $(\mathrm{N}=20)$} \\
\hline & Pre-test & Post-test & Pre-test & Post-test \\
\hline & $\%$ & $\%$ & $\%$ & \\
\hline Shirt & 70 & 100 & 60 & 55 \\
\hline Long Sleeves & 30 & 55 & 10 & 15 \\
\hline Hat & 55 & 100 & 55 & 50 \\
\hline Trousers & 10 & 10 & 10 & 10 \\
\hline Avoid the Sun & 15 & 50 & 20 & 15 \\
\hline Sunscreen & 40 & 100 & 55 & 50 \\
\hline
\end{tabular}

\section{Hypothesis Testing}

In order to test the hypotheses non-parametric statistics such as the Wilcoxon Matched-Pairs test and Kruskal Wallis One-way Anovas were used. These statistics were chosen for two reasons. Firstly, to be more conservative in the statistical analysis given the small sample size. Secondly, to reduce the type 1 error rate due to individual analysis of variables. The variables of interest have been analysed individually in order to see the effects between these variables.

Hypothesis 1 was that members of the treatment group would report higher levels of sun protection behaviour than they did at the start of the study. It was expected that the treatment group would report more sun protection behaviours than the control group. Results showed that this hypothesis was supported. Two types of non-parametric statistics were chosen to test this hypothesis. Firstly, the Wilcoxon Matched-Pairs test was used to assess the differences between sun protection behaviours on the pre and post-test for the treatment and control group. The results of this test can be found in Table 2. For the treatment group, sun protection behaviours such as sunscreen use, wearing a shirt or hat, and avoiding the sun increased significantly, but use of long sleeves and long trousers did not. No significant differences were found for the control group.

\section{Table 2}




\section{Wilcoxon Matched-Pairs Test on Sun Protection Behaviour Variables}

\begin{tabular}{|c|c|c|c|c|}
\hline Sun Protection Variables & Treatment & & Control & \\
\hline & Z Score & $\mathrm{p}$ & Z Score & $\mathrm{p}$ \\
\hline Shirt & -2.20 & $<.05$ & -1.00 & N.S \\
\hline Long Sleeves & -1.69 & N.S & -.53 & N.S \\
\hline Broad brimmed hat & -2.66 & $<.01$ & -1.00 & N.S \\
\hline Trousers & .00 & N.S & .00 & N.S \\
\hline $\begin{array}{l}\text { Avoid going out in the open during } \\
\text { the middle of the day }\end{array}$ & -2.07 & $<.05$ & -1.00 & \\
\hline Sunscreen & -3.06 & $<.01$ & -1.00 & \\
\hline
\end{tabular}

Secondly, Kruskal Wallis One-Way Anova's were used in order to assess differences between the treatment and control group on sun protection behaviours at pre-test. There were no significant differences detected between the treatment and control group for any of the sun-protection behaviours on the pre-test.

Significant differences however were detected between the two groups on the post-test (See Table 3). The only variable in which subject responses did not differ significantly was wearing long trousers. Overall, subjects in the treatment group reported more sun protection behaviours at the conclusion of the study than those in the control group. Percentage data provided in Table 1 provides additional support for these findings.

\section{Table 3}

Kruskal Wallis One-Way Anovas of Sun Protection Behaviours (SPBs) by Experimental Group for the Post-test

$\begin{array}{lllccc}\begin{array}{l}\text { Sun Protection } \\ \text { Behaviours }\end{array} & \text { Group } & \text { Mean Rank } & \mathrm{X}^{2} & \mathrm{df} & \mathrm{p} \\ \text { Shirt } & \begin{array}{l}\text { Treatment } \\ \text { Control }\end{array} & \begin{array}{l}16.5 \\ 24.5\end{array} & 9.75 & 1 & <.01 \\ & \text { Treatment } & 16.0 & & & \\ \text { Sleeves } & \text { Control } & 25.0 & 9.00 & 1 & <.01 \\ & \text { Treatment } & 16.0 & & & \\ \text { Hat } & \text { The } & 11.32 & 1 & <.001\end{array}$




\begin{tabular}{|c|c|c|c|c|c|}
\hline & Control & 25.0 & & & \\
\hline \multirow[t]{2}{*}{ Trousers } & Treatment & 20.5 & \multirow[t]{2}{*}{.00} & \multirow[t]{2}{*}{1} & \multirow[t]{2}{*}{ N.S } \\
\hline & Control & 20.5 & & & \\
\hline \multirow[t]{2}{*}{ Avoid the Sun } & Treatment & 17.5 & \multirow[t]{2}{*}{.38} & \multirow[t]{2}{*}{1} & \multirow[t]{2}{*}{$<.05$} \\
\hline & Control & 23.5 & & & \\
\hline \multirow[t]{2}{*}{ Sunscreen } & Treatment & 16.0 & \multirow[t]{2}{*}{11.32} & \multirow[t]{2}{*}{1} & \multirow[t]{2}{*}{$<.001$} \\
\hline & Control & 25.0 & & & \\
\hline
\end{tabular}

Hypothesis 2 stated that subjects in the treatment group would have increased knowledge about skin cancer compared to those in the control group. Wilcoxon-Matched-Pairs tests showed that significant increases occurred on knowledge variables for the treatment group (e.g. 'skin cancer is a dangerous disease', $Z=-2.37, \mathrm{p}<.05$; 'a suntanned person is more healthy', $Z=-3.18, \mathrm{p}<.01)$, but not for the control group.

Hypothesis 3 predicted that beliefs of people in the treatment group would change between the pre and post-test. The obtained results supported this prediction. Again the statistic used to test this hypothesis was the Wilcoxon Matched-Pairs Test. Significantly different responses were given before and after the intervention on 'I feel more healthy with a tan' $(\mathrm{Z}=-3.62, \mathrm{p}<.001)$, 'I take great care to avoid getting sunburnt' $(\mathrm{Z}=-3.41, \mathrm{p}<.001)$ and 'Clothing for sun protection should be provided for anyone who works outdoors' $(Z=-3.40$, $\mathrm{p}<.001)$. However, no significant differences were found for 'A suntanned person looks more healthy' or 'I find it difficult to protect myself from the sun'. In comparison, no significant differences were found for the control group.

Hypothesis 4 that the amount of sun exposure on the weekends would be significantly reduced for the treatment group but not for the control group, was supported by the data. A Wilcoxon Matched-Pairs test showed that there was a significant reduction in sun exposure on the weekend for the treatment group $(Z=-3.66, p<.001)$ but not for the control group. Additionally, there was no significant reduction in sun exposure on the weekdays for either group.

\section{Discussion}

The main purpose of this study was to assess changes in beliefs, knowledge and behaviours of outdoor workers towards skin cancer. These changes were to be achieved by introducing a 
skin cancer educational intervention. Results have predominantly shown that changes have occurred and that the intervention was effective.

\section{Sun Protection Behaviours}

Sun protection is vital in order to prevent the development of skin cancer. The need to educate outdoor workers about the dangers of ultra violet radiation, and teach sun protective behaviours, was supported by the finding that over half of the subjects who participated in this research reported that they had solar keratoses in the past. A principal aim of the study was to increase the levels of sun protection behaviours that outdoor workers practiced. This aim was supported by the results. Subjects who were exposed to the educational intervention reported more sun protection behaviours at the conclusion of the study than those who were not. Hence, it is feasible to suggest that information about skin cancer prevention appeared to be an effective tool for inducing reported behaviour change. This finding is congruent with other studies that have used skin cancer education in order to increase sun protection (Borland et al., 1991; Buller et al., 1994; Cody \& Lee, 1995).

An interesting finding relates to the fact that after the intervention all of the subjects in the treatment group reported wearing a shirt and hat and that they applied sunscreen. Perhaps this finding can be attributed to the Slip! Slop! Slap! media message. Subjects probably already possessed knowledge about sun protection from this national health promotion campaign, but required a reminder in order to re-engage in these behaviours. After the intervention, subjects in the treatment group also reported that they tried harder to avoid going out in the sun during the middle of the day.

The fact that no significant increases were found for long sleeves and trousers was not surprising. It is highly likely that this result is related to the temperature and humidity of outdoor work in the tropics. Instead of covering their arms and legs with clothing, the workers may need to apply large amounts of sunscreen on these parts of the body.

Since results showed no significant increases in sun protection behaviour for the control group, it is more likely that changes in the treatment group were due to the intervention. Additional findings support this. No significant differences were detected between the two groups prior to the intervention. However, after the intervention the 
treatment group reported significantly more sun protection behaviours than the control group. The use of trousers was the only sun protection behaviour in which the treatment and control group did not differ. Again, this may be because trousers are extremely hot when worn for long hours in the tropics.

\section{Skin Cancer Knowledge}

Understanding skin cancer and the risk that it poses is important in order to actively engage in sun protection. The current study aimed to achieve this by increasing knowledge about the disease. Significant increases in knowledge levels of subjects in the treatment group, indicated that the intervention was effective in teaching outdoor workers to recognise the effects of sun exposure, sun-tanning and skin cancer. Subjects who did not participate in the intervention, reported no significant increases in their knowledge. Hence, this comparison emphasizes the effectiveness of the skin cancer education. These results fortify those of Buller et al. (1994) who found that skin cancer education increased the knowledge that school children possessed about skin cancer.

Although the present educational intervention increased knowledge of the outdoor workers, as Arthey and Clarke (1995) note, "knowledge gains should be seen as a first step toward behaviour change, rather than as the main tool to alter behaviour." (p. 272)

\section{Beliefs About Skin Cancer}

Changes in beliefs before and after the intervention were interesting. Subjects in the treatment group reported that they felt less healthy with a tan after the intervention. They also tried harder to avoid getting sunburnt and agreed that protective clothing should be provided for outdoor workers. These results suggest that the information presented relating to tanning and sun protection influenced their beliefs about the deadly disease. Useful explanations can be provided for the fact that no significant changes occurred for variables such as 'A suntanned person looks more healthy' or 'I find it difficult to protect myself from the sun'. Firstly, beliefs about the healthiness of a tan are probably tied to society's emphasis on our physical appearance. Perhaps subjects have been conditioned to think that a tan looks healthier because of the bronzed bodies on television advertisements and magazine covers. Some research (Jones \& Leary, 1994) has found that informing people about the negative effects of tanning on appearance is effective in changing beliefs and behaviours. 


\section{Sun Exposure}

The aim of most skin cancer educational programs is to significantly reduce sun exposure for those at risk of developing the disease. However, outdoor workers find it difficult to reduce their sun exposure during the weekdays because of their job requirements. For this reason it was hypothesised that the treatment group would reduce their exposure to the sun on the weekend but not on the weekdays. The fact that this hypothesis was supported by the results suggests that sun exposure during the week is probably work-related. However, on the weekend in their own time subjects reported spending significantly less time in the sun. Subjects in the control group did not report a reduction in exposure to the sun which indicates that the educational intervention influenced the behaviour reduction of the treatment group. This finding provides an avenue for future research into the motivational factors of sun exposure i.e. whether it is influenced by work or pleasure.

\section{Conclusion}

As there has been limited information collated about skin cancer attitudes and behaviours of those who live in the tropics, and in particular for outdoor workers in the region, the results obtained from this study, have proved very useful. The study has shown that skin cancer education appears to be an effective tool to get outdoor workers to increase their sun protection behaviour. At the same time it has identified problem areas in sun protection (trousers, long sleeves) that may need to be addressed by health professionals in the future.

Future research should aim to include some type of observation or surveillance of sun protection behaviour. Participants may be asked to maintain a diary across the course of the study to record their daily sun protection or they may be directly observed. This would strengthen self-report measures of behaviour change by guarding against socially desirable responses. Researchers could combine self-report information with observation data and determine whether subjects are actually doing what they are reporting.

In addition, it would be useful to investigate the long-term effectiveness of a sun safety educational intervention. A second post-test using the questionnaire may be given several months after the intervention, in order to assess how much information has been retained long-term by subjects. The intervention could also be altered slightly to include information on what skin cancer is capable of doing to a person's physical appearance. Multi-media may 
be an effective tool for conveying this message. Slides showing skin cancers and the effects of their removal on appearance, could lead to improvements in sun protection behaviour. Integrating appearance-based messages with the intervention used in the present study, could perhaps improve the effectiveness of skin cancer education.

A promising future research topic would be to examine the beliefs, knowledge and behaviours towards skin cancer amongst those who spend time in the sun for recreational purposes, for example, sporting teams, bushwalkers and voluntary lifesavers. By conducting research of this nature, useful comparisons could be made between those who are paid, and those not paid to spend time in the sun. Hence, motivational factors behind sun exposure may be unveiled.

Research on knowledge about skin cancer, sun tanning attitudes and sun-protective behaviour makes a valuable contribution to the ever expanding wealth of knowledge that already exists on skin disorders and cancer. With each additional fragment of knowledge, comes hope for improved detection and prevention of this life threatening disease.

\section{References}

Arthey, S. \& Clarke, V. (1995). Suntanning and sun protection: A review of the psychological literature. Social Science Medicine, 40(2), 265-274.

Australian Institute of Health> and Welfare (AIHW) (1998). Australia's health 1998:The sixth biennial health report of the Australian Institute of Health and Welfare.

Canberra:Australian Institute of Health and Welfare.

Borland, R. Skin cancer. In Baum, A., Newman, S., Weinman, J., West, R. \& McManus, C. (Eds.), (1997). Cambridge handbook of psychology, health \& medicine. Cambridge: Cambridge University Press.

Borland, R., Hill, D. \& Noy, S. (1990). Being sunsmart: Changes in community awareness and reported behaviour following a primary prevention program for skin cancer control. Behaviour Change, 7(3), 126-135. 
Borland, R., Hocking, B., Godkin, G., Gibbs, A. \& Hill, D. (1991). The impact of a skin cancer control education package for outdoor workers. The Medical Journal of Australia, $154,686-688$.

Buller, M., Loescher, L. \& Buller, D. (1994). Sunshine and skin health. Journal of Cancer Education, 9, 155-162.

Cody, R. \& Lee, C. (1990). Behaviours, beliefs, and intentions in skin cancer revention. Journal of Behavioural Medicine, 13(4), 373-389.

Emmett, A. (1988). The bare facts: The effect of sun on skin. Sydney: Williams \& Wilkins. English, D. (1996). Biological markers of skin damage. Cancer Forum, 20(3), 215-218. Hill, D., White, V., Marks, R. \& Borland, R. (1993). Changes in sun-related attitudes and behaviours and reduced sunburn prevalence in a population at high risk of melanoma. European Journal of Cancer Prevention, 2, 447-456.

Jones, J. \& Leary, M. (1994). Effects of appearance-based admonitions against sun exposure on tanning intentions in young adults. Health Psychology, 13(1), 86-90.

Marks, R.(1996). The use of sunscreens in the prevention of skin cancer. Cancer Forum, 20(3), 211-214.

National Health and Medical Research Council.(1996). Primary prevention of skin cancer in Australia. Canberra: Australian Government Publishing Service.

Queensland Cancer Fund. (1998). Be sunsmart: Save your skin(info sheet). Brisbane: Queensland Cancer Fund.

Queensland Cancer Fund (1997a). Understanding sunspots and skin cancer. Brisbane, Queensland Cancer Fund.

Queensland Cancer Fund. (1997b). Working towards a sunsmart Queensland. Brisbane, Queensland Cancer Fund. 